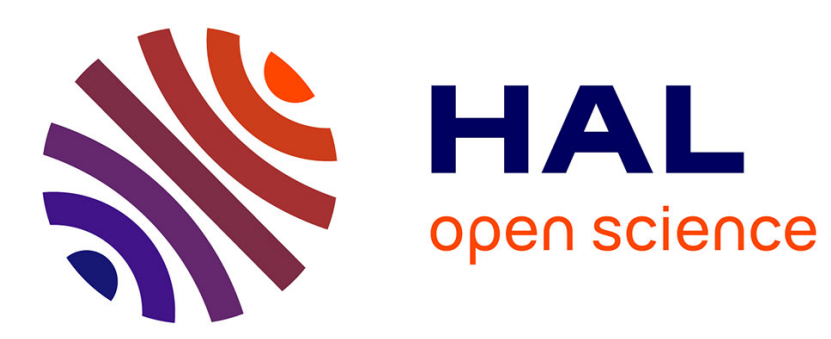

\title{
Étude numérique et expérimentale de l'échauffement d'un conducteur de forme complexe
}

\author{
J.P. Alary, C. Jablon
}

\section{To cite this version:}

J.P. Alary, C. Jablon. Étude numérique et expérimentale de l'échauffement d'un conducteur de forme complexe. Revue de Physique Appliquée, 1976, 11 (4), pp.483-487. 10.1051/rphysap:01976001104048300 . jpa-00244083

\section{HAL Id: jpa-00244083 https://hal.science/jpa-00244083}

Submitted on 1 Jan 1976

HAL is a multi-disciplinary open access archive for the deposit and dissemination of scientific research documents, whether they are published or not. The documents may come from teaching and research institutions in France or abroad, or from public or private research centers.
L'archive ouverte pluridisciplinaire HAL, est destinée au dépôt et à la diffusion de documents scientifiques de niveau recherche, publiés ou non, émanant des établissements d'enseignement et de recherche français ou étrangers, des laboratoires publics ou privés. 


\title{
ÉTUDE NUMÉRIQUE ET EXPÉRIMENTALE DE L'ÉCHAUFFEMENT D'UN CONDUCTEUR DE FORME COMPLEXE
}

\author{
J. P. ALARY et C. JABLON \\ Laboratoire de Physique des Plasmas, groupe électrotechnique et fusion contrôlée \\ Université de Paris XI, 91045 Orsay, France \\ (Reçu le 13 novembre 1975, accepté le 5 février 1976)
}

\begin{abstract}
Résumé. - On étudie numériquement l'échauffement Joule d'un conducteur inhomogène, parcouru par un courant dependant du temps en tenant compte de la variation de la résistivité avec la température et de la diffusion thermique. Le modèle numérique bidimensionnel calcule de façon cohérente les répartitions de température et de courant au cours du temps. Une expérience simple a montré la validité du modèle et un exemple d'exploitation est présenté.
\end{abstract}

\begin{abstract}
The Joule heating of an inhomogeneous, temperature dependent resistivity conductor is studied numerically when a time-varying current flows through it. The bidimensionnal numerical model computes the temperature and current distributions at each time step consistently. A simple experimental verification is presented, together with one example showing how the model may be exploited.
\end{abstract}

1. Introduction. - Les sources délivrant des énergies élevées en des temps très courts ont été jusqu'à présent constituées de bancs de condensateurs. L'énergie ainsi disponible est toutefois limitée par des questions de coût et d'encombrement à des valeurs de quelques mégajoules au plus; le besoin d'énergies nominales nettement supérieures (par exemple pour les expériences de fusion thermonucléaire contrôlée) conduit à étudier des sources différentes, utilisant le stockage inductif.

Dans ce dispositif, l'énergie est stockée magnétiquement à l'aide d'un courant de l'ordre du mégaampère circulant dans une bobine pendant un temps assez long (quelques dixièmes de secondes). Pour les applications envisagées, il est nécessaire de développer un disjoncteur rapide permettant le transfert de l'énergie vers la charge d'utilisation en des temps beaucoup plus courts.

Les disjoncteurs étudiés peuvent s'ouvrir soit par fusion d'une partie du circuit sous l'action de l'échauffement Joule [6] soit par une action mécanique [7]. Dans les deux cas, la partie rompue formant disjoncteur consiste en un étranglement de la section du conducteur, afin de minimiser l'énergie absorbée par la rupture. L'échauffement du conducteur en présence de diffusion thermique (liée à la variation de section) doit être évalué précisément pour déterminer les performances du fusible, ou au contraire éviter une élévation de température prématurée trop importante du conducteur avant sa rupture mécanique.

Nous avons à cet effet réalisé un modèle numérique de diffusion thermique instationnaire décrivant l'échauffement du conducteur représenté sur la figure 1. Nous indiquons ici successivement la mise en équation du problème $(\S 2)$, le modèle numérique qui a été réalisé (\$3), sa validation à l'aide d'expériences simples (§ 4), et enfin quelques résultats d'exploitation concernant l'échauffement du conducteur étudié pour les transferts à haute énergie ( $(5)$.

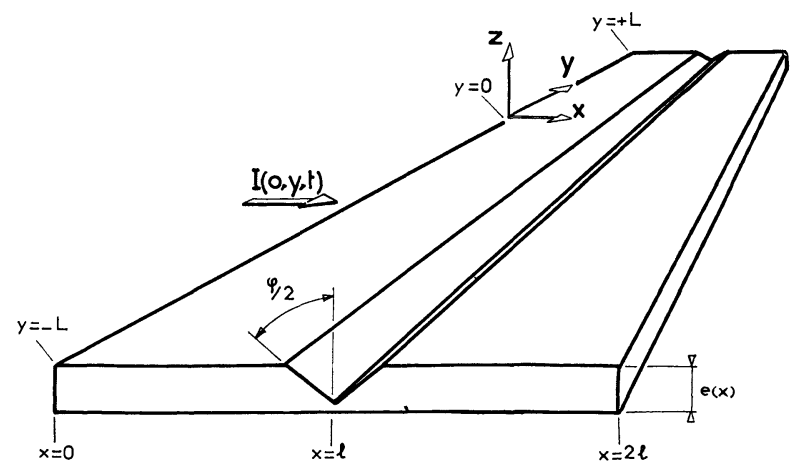

Fig. 1. - Disposition générale de l'expérience modélisée ; la densité de courant $i(O, y, t)$ est supposée connue.

2. Mise en équation du problème. - On s'attend à ce que les grandeurs physiques étudiées varient fortement en fonction de $x$ (à cause de la variation de section) et dans une moindre mesure en fonction de $y$ (en raison de gradients thermiques transverses dus à l'inhomogénéité spatiale du courant). La dépendance par rapport à la coordonnée $z$ est négligée car l'épaisseur $e(x)$ est très inférieure aux autres dimensions. 
Soient $T(x, y, t)$ et $V(x, y, t)$ la température et le potentiel électrique au point $(x, y)$ au temps $t$. Le problème est décrit par les équations suivantes :

$$
\begin{gathered}
a \nabla[e(x) \nabla T]=e(x) \partial_{t} T+H(x, y, t)+ \\
+G(x, y, t)\left[T(x, y, t)-T_{0}\right] \\
\nabla[e(x) \sigma(x, y, t) \nabla V]=0
\end{gathered}
$$

avec les conditions aux limites suivantes :

$$
\begin{gathered}
T(x, y, 0)=T_{0} \\
T(0, y, t)=T_{0} \\
\partial_{x} T(l, y, t)=0=\partial_{y} T(x, 0, t) \\
\partial_{y} T(x, L, t)=h\left[T(x, L, t)-T_{0}\right] \\
\text { (échange par convection connu) } \\
V(l, y, t)=0 \quad \text { (par convention) } \\
\partial_{y} V(x, 0, t)=\partial_{y} V(x, L, t)=0 \\
\partial_{x} V(0, y, t)=-i(0, y, t) / \sigma(0, y, t) .
\end{gathered}
$$

La composante suivant $x$ de la densité de courant $i(0, y, t)$ est imposée par le milieu extérieur et connue. Les éq. (1) et (2) sont couplées entre elles par les relations de définition suivantes :

$$
\begin{gathered}
i(x, y, t) \equiv \sigma \partial_{x} V \\
j(x, y, t) \equiv \sigma \partial_{y} V \\
\sigma=\sigma(x, y, t)=\sigma_{0} /\left\{1+\alpha\left[T(x, y, t)-T_{0}\right]\right\} \\
H(x, y, t)=\left(i^{2}+j^{2}\right) e(x) / \rho c \sigma_{0} \\
G=\alpha H-2 h / \rho c .
\end{gathered}
$$

Dans ces équations, $\rho$ est la masse volumique du matériau, $c$ sa chaleur massique spécifique, $h$ le coefficient d'échange thermique surfacique, a la diffusivité thermique, $\sigma_{0}$ la conductivité électrique à la température initiale $T_{0}$ et $\alpha$ le coefficient de variation linéaire de la conductivité $\sigma$ avec la température.

Nous avons donc réduit le problème à la résolution de deux équations aux dérivées partielles linéaires, l'une parabolique (1) et l'autre elliptique (2), couplées entre elles non linéairement. Dans le domaine géométrique fermé étudié, chacun des problèmes différentiels est bien posé [2].

2. Description du modèle numérique. - 3.1 CHOIX D'UNE MÉTHODE. - Nous avons utilisé pour résoudre des éq. (1) et (2) la méthode dite des directions alternées (décrite plus loin) qui présente l'avantage de pouvoir être indifféremment appliquée à des équations paraboliques telles que (1) ou elliptiques telles que (2) [3]. On remarque en effet que (2) est équivalente à l'équation suivante :

$$
\frac{\partial V}{\partial \tau}=\nabla(F(x, y, t) \nabla V)
$$

dans laquelle on recherche l'état stationnaire atteint pour de très grandes valeurs du temps numérique $\tau$. Le temps $\tau$ introduit dans (3) est indépendant du temps $t$ d'évolution du système modélisé. On peut également interpréter (3) en remarquant que les deux grandeurs $T$ et $V$ obéissent à des équations de diffusion, mais que le temps caractéristique de diffusion du potentiel électrostatique (défini par exemple par l'effet de peau) est très inférieur au temps d'évolution des autres phénomènes tels que la diffusion thermique et la variation de densité de courant imposée $i(0, y, t)$.

Nous rappelons donc la méthode de résolution numérique pour l'équation modèle suivante :

$\frac{\partial U}{\partial t}=\nabla(A(x, y, t) \nabla U)+B(x, y, t) U+C(x, y, t)$

qui contient comme cas particulier les éq. (1) et (2).

3.2 DiscrétisAtion DU PROBlème. - L'éq. (4) se réécrit sous la forme suivante :

$$
\begin{aligned}
\partial_{t} U & =D_{x} U+D_{y} U+S \\
D_{x} & =A \partial_{x x}^{2}+\partial_{x} A \partial_{x} \\
D_{y} & =A \partial_{y y}^{2}+\partial_{y} A \partial_{y} .
\end{aligned}
$$

Posons :

$$
\begin{array}{cl}
t=n \Delta t & n=0,1, \ldots \\
x=i \Delta x & i=0,1, \ldots, I \\
y=j \Delta y & j=0,1, \ldots, J \\
U(x, y, t)=U_{i, j}^{n} .
\end{array}
$$

Les opérateurs différentiels tels que $\partial_{x}$ et $\partial_{x x}^{2}$ sont alors modélisés par :

$$
\begin{aligned}
\partial_{x} U & \rightarrow \delta_{x} U=(2 \Delta x)^{-1}\left(U_{i+1, j}-U_{i-1, j}\right) \\
\partial_{x x}^{2} U \rightarrow \delta_{x x}^{2} U & =(\Delta x)^{-2}\left(U_{i+1, j}-2 U_{i, j}+U_{i-1, j}\right) .
\end{aligned}
$$

La méthode des directions alternées permet le calcul de $U^{n+1}$ à partir de $U^{n}$ en introduisant une valeur intermédiaire $U^{*}$, qui vérifie le système d'équations suivant :

$$
\left(U^{*}-U^{n}\right)(2 \Delta t)^{-1}=D_{x} U^{*}+D_{y} U^{n}+B U^{*}+C
$$

$\left(U^{n+1}-U^{*}\right)(2 \Delta t)^{-1}=D_{x} U^{*}+D_{y} U^{n+1}+B U^{*}+C$.

Les grandeurs $A, B$ et $C$ et les conditions aux limites qui dépendent du temps doivent dans ces équations être calculées au temps [4]

$$
t^{n+1 / 2}=t^{n}+\frac{1}{2} \Delta t .
$$

Le système (5) est un système linéaire tridiagonal facilement inversible par élimination de Gauss [3].

3.3 INCRÉMENT DE TEMPS. - La méthode des directions alternées est stable pour toutes les valeurs de l'incrément dans le temps $\Delta t$; celui-ci peut donc être choisi en fonction des variations au cours du temps des grandeurs physiques telles que la température. En pratique, nous comparons les valeurs $U^{*}$ et

$$
U^{n+1 / 2}=\left(U^{n}+U^{n+1}\right) / 2
$$


et nous choisissons un pas tel que :

$$
\operatorname{Max}\left|\frac{U^{*}-U^{n+1 / 2}}{U^{n}}\right|<\varepsilon
$$

où $\varepsilon$ mesure la précision du modèle (typiquement $\varepsilon=10^{-3}$ ).

3.4 INCRÉMENTS D'ESPACE. - Les intervalles de discrétisation spatiaux $\Delta x$ et $\Delta y$ doivent en principe être inférieurs aux longueurs caractéristiques de l'expérience, soit ici à la largeur de la rainure (voir Fig. 1). Le fait d'utiliser un maillage régulier est donc gênant, puisqu'il conduit à des points inutilement resserrés dans la quasi-totalité de la plaque. Pour pallier cet inconvénient, nous effectuons le changement de variables suivant :

$$
\begin{aligned}
& x=x(X) \quad 0 \leqslant X \leqslant 1 \quad 0 \leqslant x \leqslant l \\
& y=y(Y) \quad 0 \leqslant Y \leqslant L / l \quad 0 \leqslant y \leqslant L
\end{aligned}
$$

où les fonctions $x(X)$ et $y(Y)$ sont choisies de façon à créer, pour des valeurs équidistantes de $X$ et $Y$, un réseau convenable. Par exemple la transformation

$$
x=l \sin (k \pi X)
$$

où $k$ est un paramètre inférieur à 0,5 est particulièrement commode.

Le changement de variables conduit à une équation de forme identique à (4) pour les variables $X$ et $Y$; quoique plus complexe, cette équation peut être traitée par la méthode des directions alternées.

Cet artifice permet une adaptation du modèle numérique au problème physique comparable à des méthodes par éléments finis [5], tout en conservant la simplicité de programmation caractéristique des méthodes par différences finies à pas réguliers.

Nous avons vérifié enfin que les résultats physiques obtenus ne dépendaient pas significativement du changement de variable [6] utilisé.

3.5. Couplage NON LiNÉAIRE. - Le calcul de la température $T^{n+1}$ à partir de $T^{n}$ nécessite la connaissance des termes de source Joule au temps

$$
t^{n-1 / 2}=t_{n}+\frac{1}{2} \Delta \tau
$$

Ces termes dépendent de la source de courant imposée $i\left(0, y, t^{n+1 / 2}\right)$ qui est connue, mais également des valeurs de la conductivité $\sigma^{n+1 / 2}$, qui dépend à son tour de la température $T^{n+1 / 2}$. Une première approximation consiste à calculer les termes en question à l'aide de $\sigma^{n}$, connue, puis à effectuer une ou plusieurs itérations pour affiner le calcul de $\sigma^{n+1 / 2}$. En pratique, l'utilisation de plus d'une itération de correction n'apporte aucun changement significatif aux résultats physiques obtenus, pour des valeurs de $\varepsilon$ utilisées $\left(10^{-3}\right)$. Il appartient évidemment de déterminer dans chaque cas traité le nombre approprié d'itérations non linéaires à appliquer.
4. Validation expérimentale du modèle numérique. Le modèle réalisé suivant les principes énoncés plus haut a été soumis à un certain nombre de tests numériques visant à en assurer l'exactitude (comparaison avec une solution analytique, relations de conservations...). Il a paru intéressant d'effectuer une validation indépendante, en traitant numériquement une expérience simple et en comparant les résultats fournis par le modèle à ceux qui sont observés. Le bon accord obtenu, joint aux tests de cohérence interne du modèle garantit l'exactitude des résultats d'exploitation présentés au paragraphe 5 .

4.1 DESCRIPTION DE L'EXPÉRIENCE. - On mesure l'échauffement par effet Joule de l'éprouvette en cuivre présentée sur la figure $2 a$. Trois éprouvettes ont été réalisées avec un angle d'ouverture $\varphi$ de $30^{\circ}, 60^{\circ}$ et $90^{\circ}$; l'étranglement maximal est toujours arrondi par l'usinage, ce qui justifie l'utilisation des conditions aux limites (2a). Chaque éprouvette est percée (Fig. 2b) de

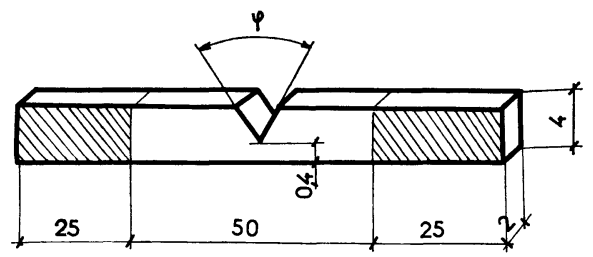

(a)

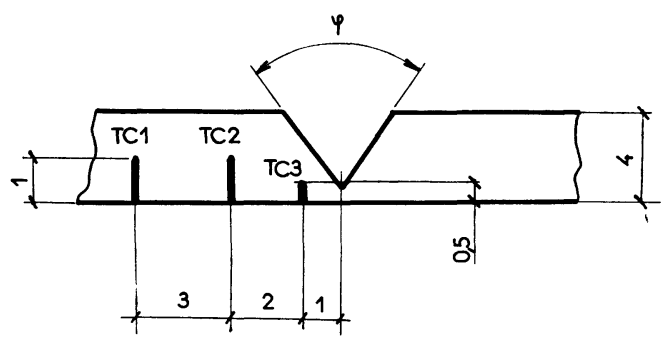

(b)

Fig. 2. - Expérience de validation; a) éprouvette utilisée (cuivre ou acier) ; $b$ ) disposition des thermocouples TC1, TC2 et TC3.

trois trous de $0,3 \mathrm{~mm}$ de diamètre et de $1 \mathrm{~mm}$ de profondeur, destinés à recevoir les thermocouples de mesure (thermocoax 2 A B A C 025 T I). Grâce à leur petite taille, ceux-ci ont un temps de réponse de $12 \mathrm{~ms}$, très inférieur au temps caractéristique de variation des températures dans l'expérience.

L'éprouvette est soumise pendant quelques secondes à un courant de l'ordre de $500 \mathrm{~A}$; on enregistre pendant chaque tir le courant instantané traversant l'éprouvette et les températures fournies par les trois thermocouples.

4.2 COMPARAISONS DU MODÈLE AVEC L'EXPÉRIENCE. - On admet que les thermocouples ont une section assez faible pour ne pas influencer les répartitions de courant et de température ; on admet également que la 
densité de courant fournie $i(O, y, t)$ est uniforme, c'està-dire indépendante de $y$, ce qui est certainement le cas pour les éprouvettes étroites qui sont utilisées. La figure 3 montre l'excellent accord entre les prévisions du modèle et les valeurs observées pour une expérience typique $\left(\varphi=90^{\circ}\right)$.

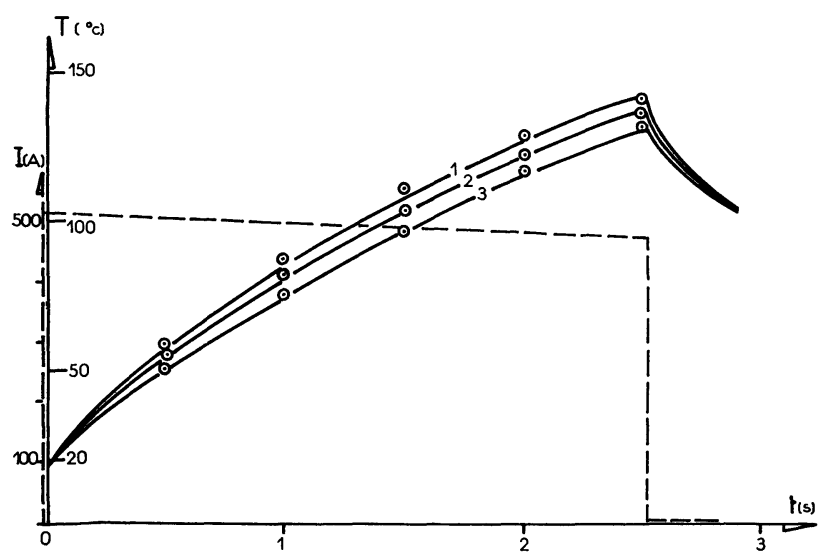

FIG. 3. - Comparaison du modèle numérique avec l'expérience. Les températures mesurées en TC1, TC2 et TC 3 sont indiquées. par des $\square$; les températures calculées par le modèle numérique figurent en trait plein et le courant total mesuré en tireté.

Les valeurs suivantes des paramètres physiques du cuivre ont été utilisées pour la comparaison entre le modèle numérique et l'expérience :

$$
\begin{aligned}
\lambda & =385 \mathrm{~W} / \mathrm{m}^{\circ} \mathrm{C} \\
a & =1,12 \times 10^{-4} \mathrm{~m}^{2} /{ }^{\circ} \mathrm{C} \\
h & =1-10 \mathrm{~W} / \mathrm{m}^{2}{ }^{\circ} \mathrm{C} \\
\sigma_{0} & =\sigma\left(20{ }^{\circ} \mathrm{C}\right)=4,2 \times 10^{7} \mathrm{mho} / \mathrm{m} \\
\alpha & =4,5 \times 10^{-3} \Omega \mathrm{m} /{ }^{\circ} \mathrm{C} .
\end{aligned}
$$

La conductivité $\sigma_{0}$ a été mesurée directement sur l'échantillon, qui n'était pas un cuivre de très bonne qualité, tandis que les autres valeurs sont les valeurs correspondants au cuivre pur. A l'exception du coefficient d'échange $h$, dont le rôle est ici négligeable, l'accord entre les résultats numériques et expérimentaux dépend assez significativement des valeurs choisies.

C'est en particulier l'impossibilité d'obtenir un accord satisfaisant avec la valeur de $\sigma_{0}$ correspondant au cuivre pur qui nous a incité à mesurer directement la conductivité de l'échantillon.

5. Exemple d'exploitation. - Nous présentons quelques résultats obtenus en modélisant un fusible destiné à être couplé à une génératrice impulsionnelle de forte énergie. Il s'agit d'un fusible en cuivre pur dont les paramètres géométriques définis sur la figure 1 sont les suivants :

$$
\begin{aligned}
L & =200 \mathrm{~mm} \\
l & =25 \mathrm{~mm} \\
\varphi & =45^{\circ} \\
e(O) & =5 \mathrm{~mm} \\
e(l) & =0,5 \mathrm{~mm} .
\end{aligned}
$$

La composante normale de la densité de courant $i(O, y, t)$ est supposée imposée par la génératrice et connue. Le calcul exact de cette fonction nécessite une description complète du circuit constitué par la génératrice, les conducteurs de raccord et le fusible. Toutefois, diverses études indépendantes nous fournissent l'évaluation raisonnable suivante $[1,6]$ :

$$
i(O, y, t)=j(y) / \operatorname{ch}\left[A\left(t_{\mathrm{M}}-t\right)\right] .
$$

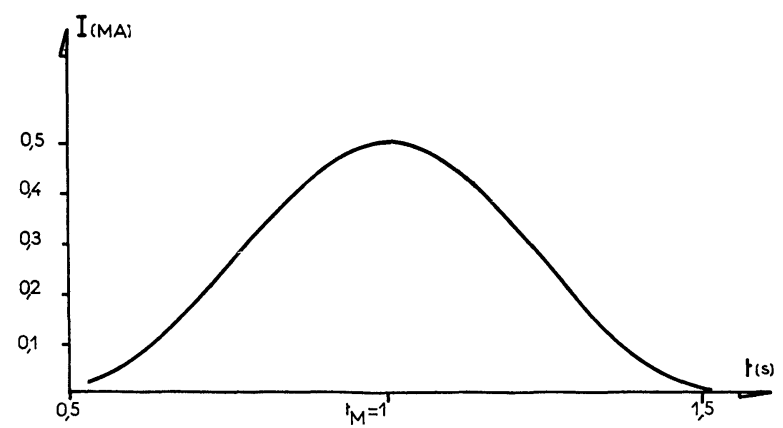

Fig. 4. - Variation temporelle du courant total modélisant celui fourni par la génératrice.

Le coefficient $A$ dépend du courant maximal imposé et $t_{M}$ est l'instant où ce courant est atteint. La figure 4 indique la variation temporelle du courant total traversant la plaque :

$$
I(t)=\int_{-L}^{L} \mathrm{~d} y e(O) i(O, y, t)
$$

dans le cas traité numériquement. La répartition $\mathrm{du}$ courant $j(y)$ choisie est indiquée sur la figure $5 a$ et les figures $5 b$ et $5 c$ représentent les équipotentielles et les

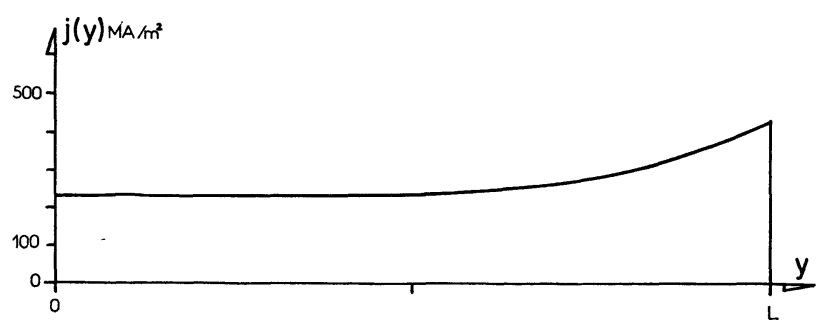

(a)

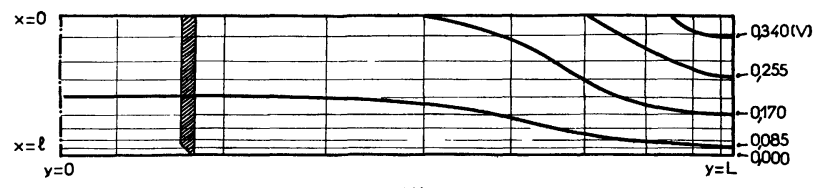

(b)

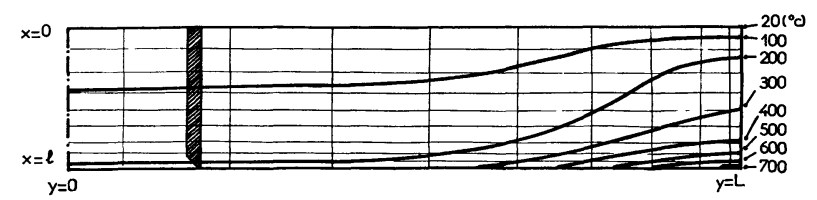

(c)

Fig. 5. - Résultats numériques à l'instant du courant maximal ; a) répartition spatiale de la densité de courant imposée au bord ; $b$ ) équipotentielles ; $c$ ) isothermes. 
isothermes à l'instant $t=t_{\mathrm{M}}$ telles que le fournit le modèle numérique. Dans cette expérience numérique, le courant total maximal de $0,5 \mathrm{~mA}$, était insuffisant pour faire fondre le fusible. On remarque l'importance de la répartition du courant $j(y)$ et surtout de la diffusion thermique transverse dans l'étranglement.

On voit l'intérêt du modèle, qui permet d'obtenir une description très détaillée du déroulement de l'expérience, avec une excellente résolution dans le temps et dans l'espace. A titre indicatif, le maillage utilisé comporte $50 \times 20$ points répartis de façon inhomogène, avec un resserrement très important vers la partie physiquement intéressante $(x=l, y=L)$; un temps de calcul de 1 min. 30 s environ sur Univac 1110 a été nécessaire pour la description complète de l'expérience, avec une conservation du flux de chaleur réalisée à mieux de $10^{-3}$ près en valeur relative.
Le modèle peut être immédiatement étendu à des problèmes où les variations de section sont beaucoup plus complexes que celles qui ont été traitées ici. Toutefois, les changements de variables permettant de suivre au mieux la géométrie du problème, doivent être de la forme (6), afin d'éviter des complications de programmation qui ôteraient à la méthode son intérêt.

En conclusion, nous avons étudié numériquement l'échauffement par effet Joule d'une plaque conductrice inhomogène.

Nous avons mis au point un modèle numérique simple, mais représentant le problème de façon assez précise et nous l'avons validé par une comparaison expérimentale. L'exploitation systématique du modèle réalisé permet de représenter des phénomènes physiques qui seraient difficilement accessibles à des mesures détaillées et de mieux les comprendre.

\section{Bibliographie}

[1] Guillet, R et al., Revue Phys. Appl. 8 (1973) 53.

[2] Morse, P. M. et Feshbach, H., Methods of theoretical physics, (McGraw Hill), 1953 p. 692.

[3] Von RosenberG, D. U., Methods for the numerical solution of partial differential equations, Elsevier, 1969.

[4] Mrtchell, J., Computational methods in partial differential equations, (Wiley), 1969.

[5] ZIENKIEWICZ, O., The finite-element method in engineering science (McGraw Hill), 1971.

[6] Geoffrion, B. et Legentil, M., Revue Phys. Appl. 10 (1975) 11.

[7] Bleys, C., Rev. Sci. Instrum. 46 (1975) 1542. 